\title{
Minimum bias and early QCD physics at LHC
}

\author{
Stefan Tapprogge $\mathrm{e}^{* \dagger}$ \\ (on behalf of the ATLAS and CMS collaborations) \\ Institut für Physik \\ Johannes Gutenberg-Universität \\ D-55099 Mainz, Germany \\ E-mail: stefan.tapproggeduni-mainz.de
}

An overview of early measurements expected from the ATLAS and the CMS experiment on the properties of proton-proton interactions at the Large Hadron Collider (LHC) is presented. As the LHC will open a new kinematic regime, it is important to validate especially the phenomenological modelling of non-perturbative effects with data by performing with initial data detailed measurements of the basic properties of proton-proton collisions in the multi- TeV regime. Examples of possible measurements in the areas of minimum bias physics, underlying event physics and jet physics are given.

XXth Hadron Collider Physics Symposium

November 16 - 20, 2009

Evian, France

\footnotetext{
* Speaker.

${ }^{\dagger}$ Supported by the German Federal Minister for Research and Technology under contract 05H09UM1 and by the Helmholtz alliance 'Physics at the Terascale'.
} 


\section{Motivation}

The Large Hadron Collider (LHC) is going to open a new kinematic regime in high energy proton-proton collisions, to be able to search for new physics beyond the Standard Model and to find the missing piece of the Standard Model, the Higgs boson. For the hard scattering part in production processes (which are mostly governed by the strong interaction), perturbative QCD (pQCD) has been well tested. However for non-perturbative effects often only a phenomenological approach is possible. Predictions for the LHC rely on the extrapolation of models tuned to existing (lower energy) data. It is therefore of utmost importance to perform measurements of basic properties of proton-proton interactions at the LHC to validate the understanding of these processes, as these will be backgrounds to searches for new physics.

The complexity of describing proton-proton interactions includes, besides the hard scattering as described by pQCD (with higher order corrections), the parton distributions functions of the proton, the underlying event (possibility of multiple parton interactions in the same proton-proton collision), inital and final state radiation (via the related Sudakov form factors), the definition of jets and (at the highest luminosities also) the minimum bias event structure.

After comments on the LHC and the two multi-purpose experiments, ATLAS and CMS, the prospects for early measurements in three categories will be described: these include examples for minimum bias physics, for underlying event physics and for jet physics.

\section{LHC, CMS and ATLAS}

The LHC start at the end of 2009 will happen at low energies ${ }^{1}$, followed in 2010 by a long physics run with center-of-mass energies of at least $7 \mathrm{TeV}$ (with a possible ramp-up to $\sqrt{s}=8-$ $10 \mathrm{TeV}$ ). For this run, an integrated luminosity of about $200-300 \mathrm{pb}^{-1}$ is envisaged.

ATLAS [1] and CMS [2] are general purpose detectors to exploit the full physics program of the LHC. They have full azimuthal coverage and extend over most of the polar angle $\theta$. Surrounding the interaction point and the beam pipe are tracking systems consisting of silicon pixel and strip detectors (for ATLAS also a straw tube tracker). These are located inside a strong solenoidal magnetic field (with a field strength of 2 (4) T for ATLAS (CMS) respectively) with a coverage in pseudo-rapidity ${ }^{2}$ extending up to $|\eta|<2.5$. Outside the tracking system, electromagnetic and hadronic calorimeter systems are found, extending the coverage up to $|\eta|<5$. These are surrounded by dedicated muon detection systems (which in case of ATLAS contains also a separate toroidal magnet system) with a coverage of up to about $|\eta|<2.5$. Both experiments have complex multi-level trigger systems to provide the necessary reduction of the bunch crossing rate, while keeping excellent efficiency for signals of interest.

During 2009, both experiments have been extensively commissioned using the signals from muons originating from cosmic ray induced air showers.

\footnotetext{
${ }^{1}$ On the last day of the conference (Nov. 20 $0^{\text {th }}$ 2009), the first beams were circulated and three days later, first collisions at $\sqrt{s}=900 \mathrm{GeV}$ were achieved and subsequently several hundred thousand events have been recorded. In December 2009 first collisions at $2.36 \mathrm{TeV}$ were provided by the LHC.

${ }^{2}$ pseudo-rapidity $\eta=-\ln \tan \theta / 2$
} 


\section{Minimum bias physics}

Traditionally, the so called 'minimum bias' (MB) events have been defined as non-single diffractive inelastic events, driven by the trigger and experimental selection criteria. Further processes which contribute to the total proton-proton cross-section are: single diffraction and elastic scattering. Even basic properties (such as the charged particle multiplicity of inelastic protonproton interactions) are uncertain at the multi-TeV energies of the $\mathrm{LHC}$, due to the extrapolation of phenomenological models from existing lower energy data. Besides providing additional information on the energy dependence of these phenomenological approaches, a precise knowledge of the properties of inelastic events is also mandatory for the design luminosity of the LHC, where on average more than 20 of those events will occur in a single bunch crossing. In the measurement, especially at low luminosity, care has to be given to possible contributions from background due to beam-gas and beam-halo events.

\subsection{Observables and measurement techniques}

Several observables can be studied using charged particles. Examples include the $\eta$ dependence of the charged particle multiplicity, the charged particle transverse momentum spectrum as well as the dependence of the average charged particle transverse momentum on the pseudorapidity $\eta$, all of which can be used to constrain (and refine the tuning of) models. Measurements of the neutral particle production are also possible using the calorimetry of the experiments, but will not be discussed further here.

A crucial component in minimum bias measurements is an efficient trigger selection which is as unbiased as possible. Various possibilities exist (which also allow mutual cross-checks and the determination of the trigger efficiency from data). A zero bias selection is possible with a random trigger (starting at the first trigger level), however this is rather inefficient at the lowest luminosities. At the cost of introducing trigger biases, the efficiency of such a random trigger can be enhanced, for example, using dedicated trigger scintillators (ATLAS), by the use of forward calorimeters (CMS) or with simple track selection criteria at the higher trigger levels.

For the measurement of the event properties, various approaches are available to recontruct the density (and properties) of charged particles:

hit counting: the charged particle density is estimated from the number of hits in the innermost layer(s) of the pixel system, which gives access also to very low $p_{T}$ particles. However no momentum measurement is possible and the contribution of secondary particle production has to be carefully estimated using simulations.

tracklets: charged particles are defined via the correlation of hits in two (or three) layers of the innermost tracking system, the pixel detector. This gives access to low $p_{T}$ particles, but without a momentum measurement.

tracks: charged particles are being reconstructed as complete tracks, providing a momentum measurement and yielding low rates of fake tracks. The challenge here is to extend the coverage to the low $p_{T}$ region, where most particle are produced. 

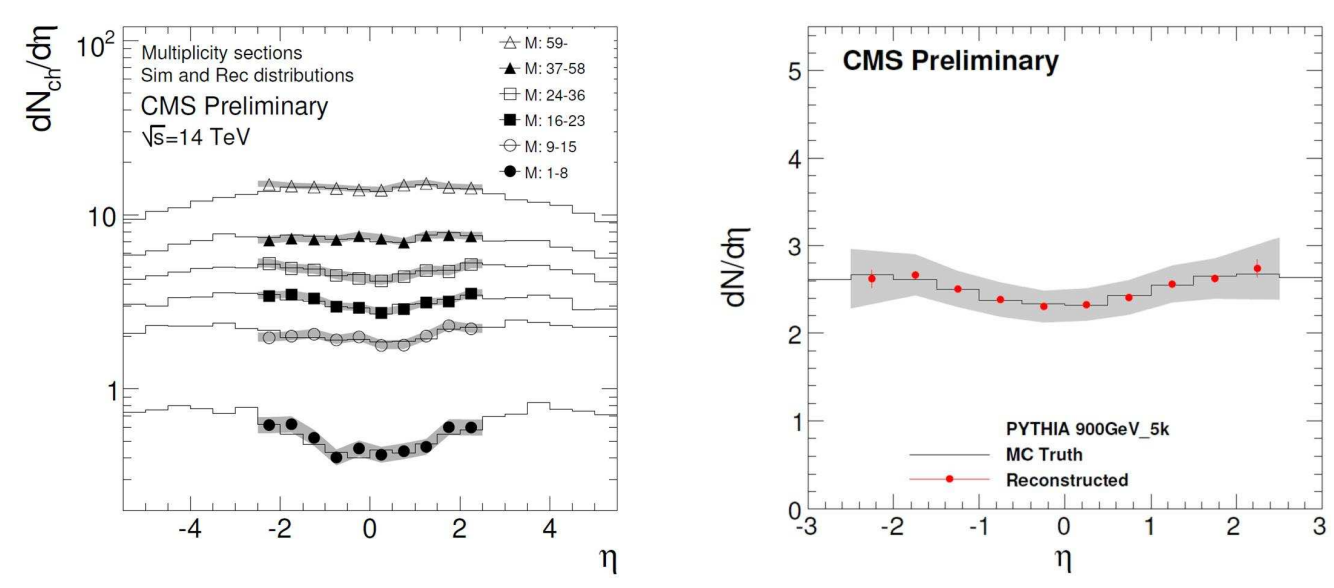

Figure 1: Left: expected distribution of $\mathrm{d} N_{c h} / \mathrm{d} \eta$ at $\sqrt{s}=14 \mathrm{TeV}$ from hit counting for several bins in the pixel hit multiplicity $M$ [纤. Right: expected distribution of $\mathrm{d} N / \mathrm{d} \eta$ at $\sqrt{s}=900 \mathrm{GeV}$ from tracklets 泡.

In all cases, the determination of the event vertex is a powerful tool to remove fake contributions and background events. It allows identification of events in which more than one proton-proton interaction has taken place in the same bunch crossing.

\subsection{Hit counting}

Particles with $p_{T}>30 \mathrm{MeV}$ reach the innermost pixel layer of CMS. Requiring a zero bias trigger and a reconstructed vertex (to calculate the pseudo-rapidity), the selection of primary pixel hits is made by a cut on the energy deposition in the silicon sensors, in order to reduce the contribution from hits due to background [ß]. A set of correction factors, derived from simulation, is then applied to take into account the impact of the event selection, of particles traversing a layer more than once (loopers) and of the mapping of hits to particles. A global systematic uncertainty of about $7 \%$ has been determined taking into account the efficiency and biases from the vertex reconstruction and the model dependence of the correction factors. The expected distribution of $\mathrm{d} N_{c h} / \mathrm{d} \eta$ (for simulation and reconstruction) is shown in Fig. 1 (left).

\subsection{Tracklets}

Tracklets are defined as pairs of hits in the first two pixel layers of the CMS experiment. For the event selection, a zero bias trigger and a reconstructed vertex (using the tracklets found) are required [Ø]. Backgrounds are subtracted using sidebands in the variable $\Delta \phi$ (used to correlate hits between the two layers), which can be done in data without simulation. Further correction factors (e.g. for efficiencies and contribution of random hits) need to be determined from simulation. The multiplicity distribution $\mathrm{d} N / \mathrm{d} \eta$ for $\sqrt{s}=900 \mathrm{GeV}$ is shown in Fig. 11 (right), based on only 5000 simulated events. The systematic uncertainty has been determined to amount to $7.5-13.5 \%$.

\subsection{Tracks}

Events are selected using the dedicated trigger scintillators of the ATLAS experiment. Charged particles are identified as well reconstructed tracks [5], with a minimum transverse momentum of $150 \mathrm{MeV}$ (using dedicated track reconstruction software). The selection also requires at least one 

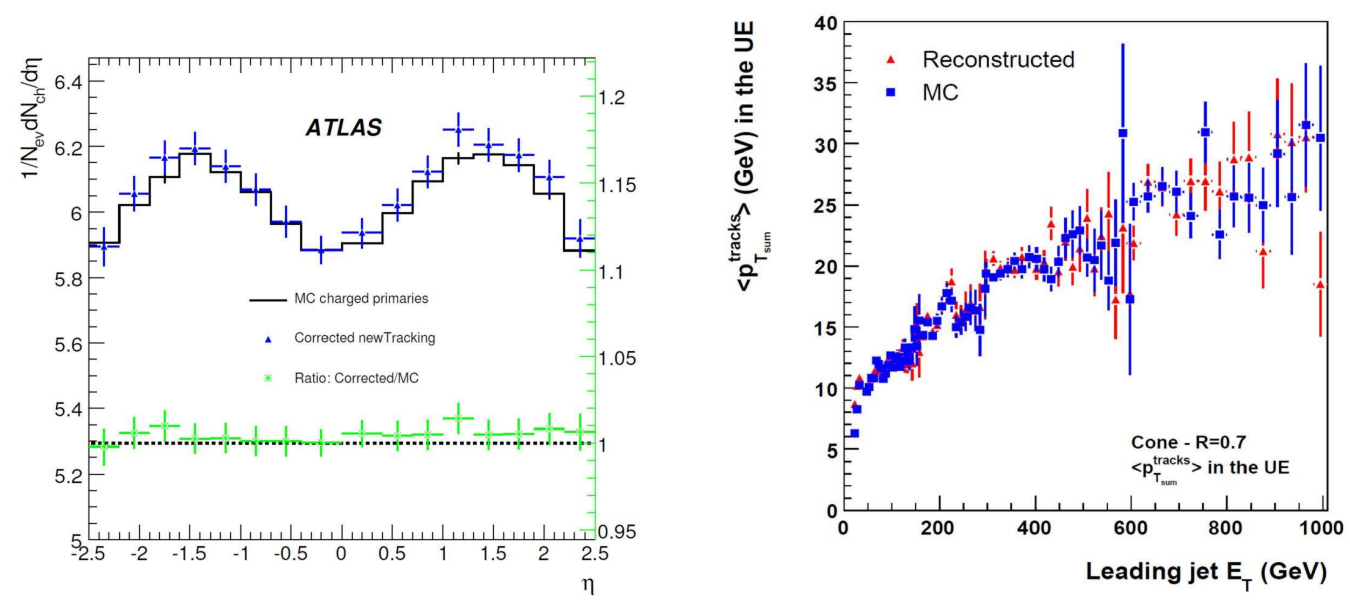

Figure 2: Left: expected distribution of $1 / N_{e v} \mathrm{~d} N_{c h} / \mathrm{d} \eta$ at $\sqrt{s}=14 \mathrm{TeV}$ for non-single diffractive events from tracks [5]. Right: average $p_{T}$ from the underlying event in the away region at $\sqrt{s}=14 \mathrm{TeV}$ [6].

reconstructed vertex. A set of corrections is applied to account for the mapping of reconstructed tracks to charged particles, for the vertex reconstruction and for the trigger bias. For the latter two, the corrections factors are applied separately on an event as well as on a per track basis. A variety of systematic uncertainties has been studied (including model dependence, selection and reconstruction efficiencies as well as backgrounds). A total systematic uncertainty of $8 \%$ has been determined. The expected multiplicity distribution for non-single diffractive events at $\sqrt{s}=14 \mathrm{TeV}$ is shown in Fig. 目(left).

\section{Underlying event physics}

The underlying event can be defined as anything happening in a proton-proton interaction besides the hard scattering process of interest. The phenomenology of the underlying event relates to multiple parton interactions and parton radiation. A thorough understanding of its properties is mandatory for precision measurements and for searches at LHC, as additional particle production can occur and influence the efficiency of selection criteria. Due to the new kinematic regime of the LHC, uncertainties exist in the predictions of models for the underlying event (taking into account that only few measurements have been made at lower energies). Measurements need to be performed in a variety of hard scattering processes, such as jet production, Drell-Yan lepton pair production and the associated production of $W(Z)$ bosons with jets.

As an example for a possible measurement of the underlying event activity, events with jets are considered by ATLAS [6]. In the plane transverse to the beam axis, three regions are defined by the difference in azimuthal angle, $\Delta \phi$, between a track and the leading jet in the event: the 'toward' region $\left(|\Delta \phi|<60^{\circ}\right)$, the 'away' region $\left(|\Delta \phi|>120^{\circ}\right)$ and the transverse region $\left(60^{\circ}<|\Delta \phi|<120^{\circ}\right)$. In the transverse region the production of charged particles is studied and the dependence of the average track transverse momentum on the $E_{T}$ of the leading jet is investigated. Fig. 目 (right) shows the expected distribution, both for generated and reconstructed events, indicating that only small corrections are needed to unfold the data. 

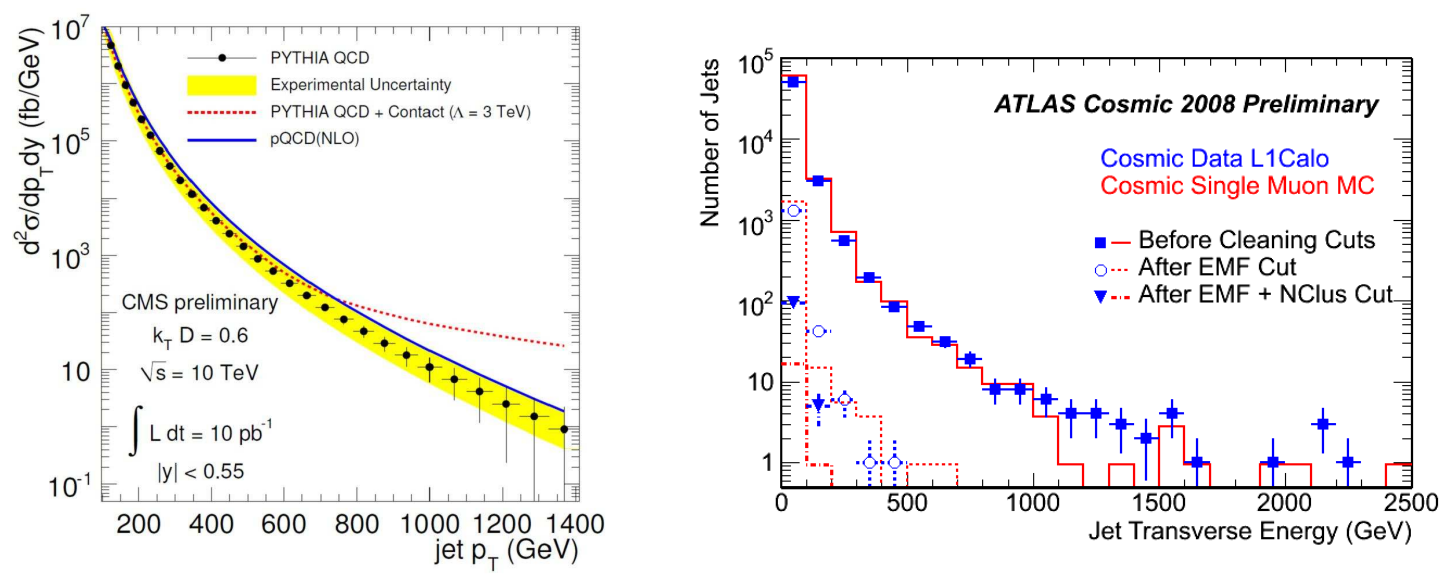

Figure 3: Left: inclusive jet cross-section at $\sqrt{s}=10 \mathrm{TeV}$ [9] with uncertainties as expected for an integrated luminosity of $10 \mathrm{pb}^{-1}$. Right: measured jet transverse energy in cosmic ray induced muon events [10].

\section{Jet physics}

Hard scattering processes with outgoing quarks or gluons manifest itself in events containing jets (bundles of particles). In contrast to leptons, jets need to be defined via a jet algorithm, which has to fulfill certain criteria (e.g. infra-red and collinear safety for proper comparison to theoretical calculations of the jet cross-section). The experimental challenge includes the determination of the true jet energy from the observed detector response, this involves the calibration of the jet energy ('energy scale') as well as the unfolding for smearing ('energy resolution').

In addition to inclusive jet production (differential cross-section as a function of $p_{T}$ ), events with di-jets allow studying the azimuthal angular distribution, the di-jet invariant mass cross-section and a triple differential cross-section. Furthermore, studies of the shape of jets are sensitive to the modelling of QCD radiation. Other observables not discussed here include the production of multijets and the exclusive production of jets reps. the one of events with (rapidity) gaps between jets.

At leading order, the two jets (partons) from a hard scatter are expected to be back-to-back in azimuthal angle $(\Delta \phi=\pi)$. Smaller values of $\Delta \phi$ (called decorrelation) are expected due to radiation effects (higher order processes and/or parton showering). As studied by ATLAS [П], the $\Delta \phi$ distribution is rather insensitive to the knowledge of the absolute energy scale, but is however sensitive to the modelling of these radiation effects. Other observables which provide sensitivity to the modelling of multi-jet events are event shape distributions, e.g. the global transverse thrust and the global thrust minor, as investigated by CMS [8]. These are insensitive to the choice of the jet algorithm and can provide input for tuning of the simulation of jet production.

In the measurement of the inclusive jet cross-section, an important ingredient is the determination of the jet energy scale, which can be done in situ (using data). The relative intercalibration of different $\eta$ regions is done using di-jet events, for the absolute energy calibration the $p_{T}$ balance in $\gamma / Z$-jet events is used. As shown in a study by CMS [ [?], the calibration of jets up to transverse momenta of $600 \mathrm{GeV}$ can be carried out with $10 \mathrm{pb}^{-1}$ of integrated luminosity at a center of mass energy of $10 \mathrm{TeV}$. For this integrated luminosity the systematic uncertainty dominates in the cross-section measurement over the statistical one up to $p_{T}<1 \mathrm{TeV}$ and is larger than the present 
theoretical uncertainties. Fig. 3 (left) shows the expected inclusive jet cross-section, from unfolded pseudo-data as well as the theoretical expectation. At the highest $p_{T}$ values, this observable has also sensitivity to new physics processes (example shown of a contact interaction model).

Muons originating from cosmic ray induced air showers can give rise (via Bremsstrahlung) to large energy deposits (of electromagnetic nature) in the ATLAS calorimeters. These backgrounds to jet production have been determined during the commissioning phase of the experiments and can be suppressed effectively [10] by cuts (e.g. on the electromagnetic energy fraction of a jet), as shown in Fig. 3 (right).

\section{Conclusion and outlook}

Using the data expected from the first physics run of the LHC in 2010 at center-of-mass energies of at least $7 \mathrm{TeV}$, a broad variety of QCD related measurements will be made by ATLAS and CMS, ranging from soft processes (e.g. properties of minimum bias events) to hard scattering processes (e.g. jet production). These early measurements are of importance in order to establish the basic properties of proton-proton interactions in this new kinematic regime. This will give further insight especially in the phenomenology of the non-perturbative part of the strong interaction (validating and allowing to adjust models) and lay the foundation of searches for new physics processes.

\section{References}

[1] G. Aad et al. (ATLAS collaboration), The ATLAS experiment at the CERN Large Hadron Collider, JINST 3 (2008) S08003.

[2] S. Chatrchyan et al. (CMS collaboration), The CMS experiment at the CERN LHC, JINST 3 (2008) S08004.

[3] CMS collaboration, Pseudorapidity distributions of charged hadrons in minimum-bias $p-p$ collisions at $\sqrt{s}=14 \mathrm{TeV}$, CMS-PAS-08-004 (2008).

[4] CMS collaboration, Study of charged hadron multiplicity in minimum-bias $p+p$ collisions at $\sqrt{s}=900 \mathrm{GeV}$ and $10 \mathrm{TeV}$, CMS-PAS-09-002 (2009).

[5] G. Aad et al. (ATLAS collaboration), Expected performance of the ATLAS experiment, detector, trigger and physics, CERN-OPEN-2008-020 (arXiv:0901:0512 [hep-ex]).

[6] A. Moraes, C. Buttar and D. Clements, Measuring the underlying event at ATLAS, ATLAS-PHYS-PUB-2005-015 (2005).

[7] A. Moraes, C. Buttar, D. Clements and I.O. Skilicorn, Preparing for measurements of dijet azimuthal decorrelations at ATLAS, ATLAS-PHYS-PUB-2006-013 (2006).

[8] CMS collaboration, Study of hadronic event-shape variables, CMS-PAS-08-003 (2008).

[9] CMS collaboration, Initial measurement of the inclusive jet cross section at $10 \mathrm{TeV}$ with CMS, CMS-PAS-08-001 (2008).

[10] ATLAS collaboration, https://twiki.cern.ch/twiki/bin/view/Atlas/ApprovedCosmicPlotsJetEtMiss (2009). 